\title{
Universal Scaling Theory of the Boundary Geometric Tensor in Disordered Metals
}

\author{
Miklós Antal Werner, ${ }^{1}$ Arne Brataas, ${ }^{2}$ Felix von Oppen, ${ }^{3}$ and Gergely Zaránd ${ }^{1}$ \\ ${ }^{1}$ Exotic Quantum Phases "Momentum" Research Group, Department of Theoretical Physics, \\ Budapest University of Technology and Economics, 1111 Budapest, Budafoki út 8, Hungary \\ ${ }^{2}$ Center for Quantum Spintronics, Department of Physics, Norwegian University of Science and Technology, \\ NO-7491 Trondheim, Norway \\ ${ }^{3}$ Dahlem Center for Complex Quantum Systems and Fachbereich Physik, Freie Universität Berlin, 14195 Berlin, Germany
}

(Received 25 July 2018; revised manuscript received 25 October 2018; published 12 March 2019)

\begin{abstract}
We investigate the finite-size scaling of the boundary quantum geometric tensor (QGT) numerically close to the Anderson localization transition in the presence of small external magnetic fields. The QGT exhibits universal scaling and reveals the crossover between the orthogonal and unitary critical states in weak random magnetic fields. The flow of the QGT near the critical points determines the critical exponents. Critical distributions of the QGT are universal and exhibit a remarkable isotropy even in a homogeneous magnetic field. We predict universal and isotropic Hall conductance fluctuations at the metal-insulator transition in an external magnetic field.
\end{abstract}

DOI: 10.1103/PhysRevLett.122.106601

Introduction.-The geometrical structure of the Hilbert space continues to receive a lot of attention. The FubiniStudy metric tensor of the Hilbert space [1,2], also referred to as Fisher information metric [2], provides a natural measure of distance in the Hilbert space, related to quantum fidelity - a fundamental concept in quantum information science [3]. The Fubini-Study metric tensor [4,5] has also been used to analyze quantum critical points in many systems including interacting spin models [6,7], manybody systems $[8,9]$, and systems exhibiting topological order $[10,11]$. Non-adiabatic dynamics in driven quantum systems is also deeply connected to the Riemannian metric of the ground state manifold [12].

Another fundamental geometric concept in quantum theory is the Berry phase [13], a geometric invariant in quantum state manifolds. The presence of a nontrivial Berry curvature leads to interesting quantum interference phenomena $[14,15]$ and impacts the trajectory of wave packets $[16,17]$. The Zak phase $[15,18]$ — the Berry-phase associated with closed loops in the Brillouin zone-and its higher dimensional analogs play a central role in the description of topological insulators [19-21]. Non-Abelian Berry phases of degenerate ground state manifolds, on the other hand [22], can generate spin relaxation in the absence of external magnetic fields [23] and underlie realizations of nonAbelian statistics in topological superconductors [24].

The concepts of the Fubini-Study metric tensor and the Berry phase can be unified through the so-called quantum geometric tensor (QGT) [25]. Consider a quantum system, whose Hamiltonian $H\left(\left\{\phi_{i}\right\}\right)$ and eigenstates $\left|\alpha\left(\left\{\boldsymbol{\phi}_{i}\right\}\right)\right\rangle$ depend smoothly on a set of real parameters, $\phi_{i}$. The QGT of the eigenstate $\left|\alpha\left(\left\{\phi_{i}\right\}\right)\right\rangle$ at a point $\left\{\phi_{i}\right\}$ in the parameter space is then defined as

$$
Q_{\alpha}^{i j}(\phi) \equiv\left\langle\partial_{\phi_{i}} \alpha \mid \partial_{\phi_{j}} \alpha\right\rangle-\left\langle\partial_{\phi_{i}} \alpha \mid \alpha\right\rangle\left\langle\alpha \mid \partial_{\phi_{j}} \alpha\right\rangle
$$

The matrix $Q_{\alpha}^{i j}$ is Hermitian and gauge invariant. Its (symmetric) real part is the metric tensor associated with the manifold $|\alpha(\phi)\rangle[1,25]$, while its imaginary (antisymmetric) part is the Berry curvature form [13,25], whose surface integral yields the Berry phase associated with closed loops in parameter space.

In the present Letter, we demonstrate that the QGT offers deep insight into a long-standing problem in condensed matter physics, Anderson's disorder-driven metal-insulator (MI) transition in small external magnetic fields [26,27]. In particular, the structure of the QGT reflects the universality class of the Anderson transition. Elements of the QGT display universal finite size scaling close to the metalinsulator transition, and capture the flow between the orthogonal $(B=0)$ and unitary $(B \neq 0)$ universality classes. At the transition, the elements of the QGT have universal distributions, characteristic of the underlying symmetry of the transition, but, surprisingly, independent of the direction of the external field. We predict that these universal fluctuations show up as universal and isotropic Hall conductance fluctuations at the metal-insulator transition.

Mathematical model.-To investigate the effect of small magnetic fields on the properties of eigenstates close to the MI transition, we study disordered noninteracting spinless fermions on a three-dimensional cubic lattice in external magnetic fields, as described by

$$
\hat{H}=\sum_{\mathbf{r}} V_{\mathbf{r}} c_{\mathbf{r}}^{\dagger} c_{\mathbf{r}}-\sum_{\left\langle\mathbf{r} \mathbf{r}^{\prime}\right\rangle}\left(t_{\mathbf{r r}^{\prime}} c_{\mathbf{r}}^{\dagger} c_{\mathbf{r}^{\prime}}+\text { H.c. }\right) \text {. }
$$

Here $c_{\mathbf{r}}^{\dagger}\left(c_{\mathbf{r}}\right)$ creates (annihilates) a fermion on site $\mathbf{r}=$ $(x, y, z)$, and the $V_{\mathbf{r}}$ denote independent random variables 
uniformly distributed in the interval $[-W / 2, W / 2]$. The second term in Eq. (2) accounts for nearest neighbor hopping, with the magnetic field incorporated through the Peierls substitution, $t_{\mathbf{r r}^{\prime}}=t e^{i 2 \pi A_{\mathrm{rr}^{\prime}}}$. In homogeneous fields, we use the gauge of Ref. [28] for the bond vector potentials $A_{\mathbf{r r}^{\prime}}$, while in random fields the $A_{\mathbf{r r}^{\prime}}$ denote independent, uniformly distributed random variables from the interval $\left[-W_{B} / 2, W_{B} / 2\right]$.

Single particle eigenstates of $\hat{H}$ are usually classified as "extended" or "localized." The latter emerge close to the band edge, and are separated from the former at small disorder by so-called mobility edges [27]. Correspondingly, the system is insulating or metallic if states at the Fermi energy are localized or extended, respectively.

Universality classes and criticality.-The spatial structure of localized (extended) states is characterized by the localization (coherence) length, $\xi$. These latter length scales depend on the energy of the states, and diverge at the mobility edge $E_{c}$ following a power law, $\xi \sim\left|E-E_{c}\right|^{-\nu}$. This divergent behavior is the ultimate basis of single parameter scaling theory [29]: assuming that close to the MI transition $\xi$ is the only relevant length scale, the zero temperature dimensionless conductance $g=G /\left(e^{2} / h\right)$ of a system of size $L$ must be a function of $L / \xi$ only, and therefore must obey the scaling equation, $\partial g / \partial \ln L=\beta(g)$, with $\beta(g)$ a universal function. This beta function has indeed been determined both perturbatively and numerically in the absence of external magnetic field, and its universal properties have been convincingly demonstrated [29-31]. In the presence of a sufficiently strong time reversal symmetry breaking, however, a clearly distinct, but apparently also universal scaling has been observed [32].

The beautiful construction of single parameter scaling must therefore necessarily break down in small magnetic fields. A weak external magnetic field generates a magnetic length scale, $L_{B}$, which is typically much larger than all microscopic length scales. As a consequence, the dimensionless conductance should also depend on the ratio $L_{B} / \xi$, implying a two-parameter dependence, $g=g\left(L / \xi, L_{B} / \xi\right)$, and invalidating the single parameter scaling theory [33]. Fortunately, very close to the transition-or in very large fields-we have $L_{B} / \xi \rightarrow 0$. Therefore universal scaling is still recovered at criticality, but with a modified "unitary" beta function, $\beta \rightarrow \tilde{\beta}(g)$ [34]. So far the intriguing crossover between orthogonal and unitary criticality has not been observed systematically in experiments, but it has been investigated to some extent within the nonlinear sigma model approach [35-37], where the crossover in a weak magnetic field has been addressed near the orthogonal critical point in $2+\varepsilon$ dimensions. Perturbative scaling gives a qualitative picture of the crossover, but the approximate values of the critical exponents are in significant disagreement with numerical results [31]. A precise description of criticality near the unitary fixed point therefore appears to be beyond the reach of this perturbative approach. An orthogonal-unitary crossover has been observed numerically in the critical level spacing statistics in Ref. [38], but the violation of the one parameter scaling theory is not addressed in that work. As we show now, the quantum geometric tensor provides an ideal tool to characterize this crossover.

Two-parameter QGT scaling theory.-In the spirit of Thouless [39], who related the boundary condition dependence of single particle energies to the dimensionless conductance, we shall investigate the boundary condition dependence of the single particle eigenstates of Eq. (2), determined by the eigenvalue equation

$$
|\alpha\rangle=\sum_{\mathbf{r}} \alpha(\mathbf{r}) c_{\mathbf{r}}^{\dagger}|0\rangle, \quad \hat{H}|\alpha\rangle=E_{\alpha}|\alpha\rangle .
$$

We prescribe here twisted boundary conditions, $\alpha_{\phi}(\mathbf{r}+L \mathbf{n})=e^{i \mathbf{n} \cdot \boldsymbol{\phi}} \alpha_{\phi}(\mathbf{r})$, with $\mathbf{n}=\left\{n_{x}, n_{y}, n_{z}\right\}$ a vector of arbitrary integers, and $\phi=\left\{\phi_{x}, \phi_{y}, \phi_{z}\right\}$ collecting the boundary twists into a single vector. For a given system size and disorder realization, we can now view each eigenstate as a manifold, $|\alpha\rangle=|\alpha(\phi)\rangle$, and define the corresponding QGT at zero twist, $Q_{\alpha}^{i j} \equiv Q_{\alpha}^{i j}(\boldsymbol{\phi}=0)$. In the presence of time reversal symmetry, the antisymmetric part of the tensor $Q_{\alpha}^{i j}$ vanishes. Moreover, the sum of the antisymmetric part of $Q_{\alpha}^{i j}$ over occupied states is the Hall conductance [40]. The antisymmetric part of the QGT is therefore a promising dimensionless indicator of time reversal symmetry breaking. Diagonal elements of $Q^{i j}$ are reminiscent of the Thouless number, and turn out to be in one to one correspondence with it in the presence of large magnetic fields as well as in their absence, though the precise functional relation is different [41].

These observations lead us to introducing two real parameters for each eigenstate $|\alpha\rangle$,

$$
g(\alpha) \equiv \operatorname{tr}\left\{Q_{\alpha}^{i j}\right\}, \quad h(\alpha) \equiv i\left(Q_{\alpha}^{x y}-Q_{\alpha}^{y x}\right) .
$$

These parameters fluctuate strongly for distinct disorder potentials and eigenstates. The power-law tail of their distribution makes usual averages ill-defined; therefore we define the "typical" magnitudes of these parameters through logarithmic averages over a large ensemble of samples [47-49],

$\ln g_{\mathrm{typ}} \equiv{\overline{\ln |g(\alpha)|_{E_{\alpha} \approx E}}}, \quad \ln h_{\mathrm{typ}} \equiv \overline{\ln |h(\alpha)|}_{E_{\alpha} \approx E}$.

Notice that $g_{\text {typ }}$ and $h_{\text {typ }}$ are both functions of energy, system size, disorder strength, and magnetic field. As we demonstrate, these quantities behave as good scaling parameters, and satisfy the universal scaling equations

$$
\frac{\partial g_{\mathrm{typ}}}{\partial \log L}=\beta_{g}\left(g_{\mathrm{typ}}, h_{\mathrm{typ}}\right), \quad \frac{\partial h_{\mathrm{typ}}}{\partial \log L}=\beta_{h}\left(g_{\mathrm{typ}}, h_{\mathrm{typ}}\right) .
$$




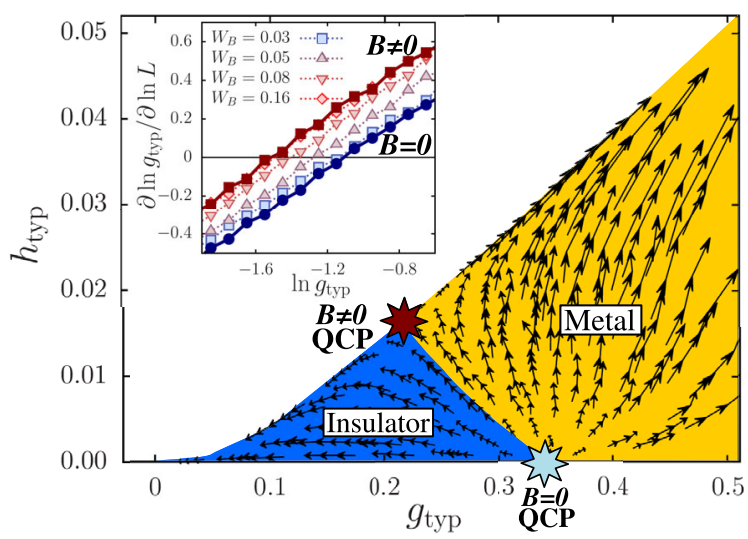

FIG. 1. Finite size scaling of $g_{\text {typ }}$ and $h_{\text {typ }}$ in a random magnetic field. Arrows indicate the direction of the renormalization group flow upon increasing the system size from $L=8$ to $L=14$. The $B=0$ (orthogonal) and $B \neq 0$ (unitary) quantum critical points are denoted by light and red stars, respectively. The blue region indicates the insulating phase, while the metallic phase is yellow. Inset: Filled symbols: universal single parameter scaling functions $\beta=L \partial_{L} \ln g_{\text {typ }}$ for $B=0$ (blue circles), and large homogeneus fields, $B \neq 0$ (red squares). Empty symbols show $L \partial_{L} \ln g_{\text {typ }}$ in weak random magnetic fields for system sizes increased from $L_{1}=8$ to $L_{2}=14$. These nonuniversal crossover curves interpolate between the orthogonal and the unitary scaling functions, and evolve into the latter upon increasing random field.

The content of Eq. (6) is that the logarithmic size dependence of the typical values $g_{\mathrm{typ}}$ and $h_{\mathrm{typ}}$ is completely independent of microscopic details such as $W, W_{B}$, or the location of the Fermi energy, and is solely determined by $g_{\mathrm{typ}}$ and $h_{\mathrm{typ}}$. These equations resemble somewhat those of Pruisken and Khmelnitskii, derived for the quantum Hall transition [50,51], and also verified numerically [28,52].

To verify the scaling hypothesis of Eq. (6), we first performed finite size computations in a homogeneous field, and evaluated the logarithmic derivatives on the left hand side for various disorder realizations and energies numerically. In the absence of external field, $h(\alpha) \equiv 0$ for each level, we recover a flow along the axis $h_{\mathrm{typ}}=0$ of the $\left(g_{\mathrm{typ}}, h_{\mathrm{typ}}\right)$ plane, as shown in Fig. 1. This flow is governed by the one-parameter function, $\beta_{g}\left(g_{\mathrm{typ}}, 0\right)$, which we determined numerically (see inset of Fig. 1). A critical point emerges at $g_{B=0}^{*}=0.3309(18)$, and the numerically determined $\beta$ function yields a critical exponent $\nu_{B=0}=$ $1.560(63)$ in good agreement with the best known result for orthogonal systems, $\nu_{\text {orth }}=1.571(8)$ [31]. We thus find that the trace of the QGT behaves as an appropriate scaling variable, which can be used to replace the dimensionless conductance of the single-parameter scaling theory of Ref. [29] or the Thouless conductance [39].

Piercing just one flux quantum through our system brings us immediately to the unitary class of systems: it yields another, universal one-parameter trajectory in the $\left(g_{\mathrm{typ}}, h_{\mathrm{typ}}\right)$ plane, with a critical point at $g_{B \neq 0}^{*}=0.22215(87)$ and a finite $h_{B \neq 0}^{*}=0.01683(13)$. Again, the extracted value of the critical exponent, $\nu_{B \neq 0}=1.459(64)$ agrees well with the most accurate estimate in the literature, $\nu_{\text {unitary }}=1.424(15)$ [53]. These results prove that the antisymmetric part $h$ of the QGT provides a good dimensionless, universal variable to distinguish the orthogonal and unitary universality classes.

Unfortunately, within the torus geometry used here, we cannot pierce less than one flux quantum through the system [28], and this is already too large to observe the flow between the two critical points in the $\left(g_{\mathrm{typ}}, h_{\mathrm{typ}}\right)$ plane. We circumvented this difficulty by applying random vector potentials. In this case, we can tune the strength of time reversal symmetry breaking continuously by changing the strength $W_{B}$ of the random vector potentials. Reassuringly, in large random fields, $W_{B} \gtrsim 0.15$, the flow perfectly coincides with the one parameter trajectory that we observed in homogeneous fields. In small random fields $\left(W_{B} \lesssim 0.15\right)$, however, we can now clearly observe a twoparameter flow crossing over between the $B=0$ and $B \neq 0$ universality classes, as presented in Fig. 1. We should emphasize that the flow in Fig. 1 is independent of microscopic details, and is generated at each point by collecting data at different energies, and for different values of the disorder parameters $W$ and $W_{B}$. Analysis of the linearized flow around the fixed points [41] also allows us to extract the exponents associated with relevant (and irrelevant) operators [54] at the critical points. At the $B=0$ critical point, the flow is characterized by the "thermal" exponent $y_{1}^{B=0}=1 / \nu_{B=0}$, and an exponent associated with the presence of random flux, $y_{2}^{B=0}=0.990(11)$. The latter agrees with the "engineering dimension" of a random flux field, $y_{\text {flux }}=1$ [55]. At the unitary critical point, the flow is governed by a relevant eigenvalue, $y_{1}^{B \neq 0}=1 / \nu_{B \neq 0}$ and an irrelevant eigenvalue, $y_{2}^{B \neq 0}=-2.12(23)$, which we can associate with the dimension of the leading irrelevant operator. Interestingly, in our tight minding model, all data points $\left(g_{\mathrm{typ}}, h_{\mathrm{typ}}\right)$ were found to lie under the unitary critical line in Fig. 1, and we were unable to reach the region above it.

Critical QGT distributions. - The typical values $g_{\mathrm{typ}}$ and $h_{\text {typ }}$ still allow for large sample to sample and level to level fluctuations of $g(\alpha)$ and $h(\alpha)$ at and around the Fermi energy, and a corresponding broad distribution. At the critical points $\left(g_{\mathrm{typ}}^{*}, h_{\mathrm{typ}}^{*}\right)$, the Fermi energy lies just on the mobility edge, $E_{C}$, where these distributions are expected to become independent of the sample size (scale invariant) and universal. To determine these universal distributions, we first locate the mobility edge for each disorder strength $W$ and $W_{B}$, and then extract the critical distributions of the quantum geometric tensor $Q_{\alpha}^{i j}$.

Figures 2(a)-2(b) show the critical distributions $p[g(\alpha)]$ and $p[h(\alpha)]$, both independent of system size, disorder, and magnetic field strength. The tail of $p(g)$ follows a power law with an exponent close to $\eta=\beta / 2+3 / 2$, with $\beta=1$ 

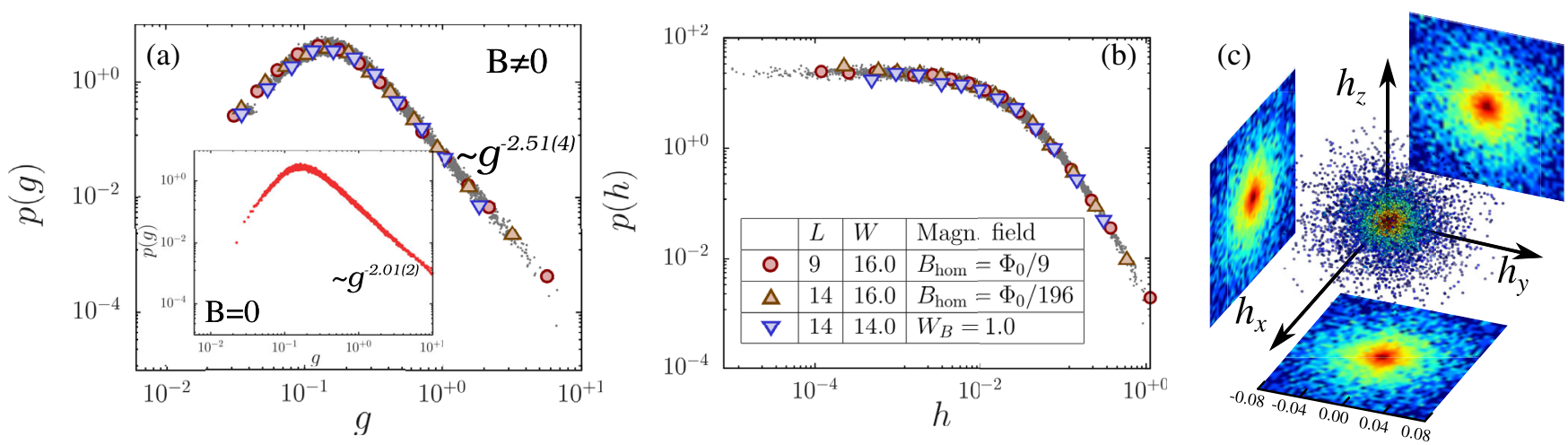

FIG. 2. (a)-(b) Probability distributions $p(g)$ and $p(h)$ at the unitary $(B \neq 0)$ critical point, extracted from systems with different system sizes, magnetic fields, and disorder strengths, with the energy at the mobility edge. The highlighted symbols refer to specific data sets (see legend), while small gray dots show distributions obtained by merging all data of all parameter sets. Inset of (a) Distribution of $g$ at the orthogonal $(B=0)$ critical point. The distributions $p(g)$ are clearly different in the two universality classes and are characterized by different exponents, too. (c) Joint distribution of the parameters $h_{x / y / z}$, characterizing the antisymmetric part of the QGT, at the unitary critical point. Points in the three-dimensional cloud represent individual eigenstates in a homogeneous magnetic field $B=\Phi_{0} / 9$ along the $z$ direction, in a system of size $L=9$ and disorder $W=17$. The almost perfect rotational symmetry of the distribution is supported by the two-dimensional marginals.

and $\beta=2$ the exponents characterizing the level spacing distribution in the orthogonal and unitary classes, respectively. (The relation $\eta=\beta / 2+3 / 2$ follows from heuristic arguments, presented in the Supplemental Material [41].) The distribution of the Thouless curvature exhibits a similar tail, but with a different exponent, $\tilde{\eta}=\beta+2$ [56-58]. Interestingly, systems with homogeneous and random fields give rise to identical distributions. This surprising agreement of the distributions in homogeneous and random fields indicates that the direction of the magnetic field is irrelevant at the critical point, at least from the point of view of the quantum geometric tensor's structure and distribution. Therefore the statistics of the QGT should be not only universal, but also rotationally invariant at the critical point.

To explore this symmetry, we generalized the parameter $h$ and characterized the antisymmetric part of $Q^{i j}$ by three independent real numbers forming an axial vector,

$$
h_{k}(\alpha) \equiv i \sum_{i, j} \epsilon_{k i j} Q_{\alpha}^{i j}
$$

with $\epsilon_{k i j}$ as the completely antisymmetric tensor. As shown in Fig. 2(c), the joint critical distribution of the three parameters $h_{x, y, z}$ shows remarkable isotropy, even in a strong homogeneous magnetic field and the typical values of $\left|h_{x, y, z}\right|$ are all equal. This observation also justifies a posteriori the somewhat arbitrary choice of $h=h_{z}$ as a scaling variable in a random field, too.

A detailed analysis of the distribution of the $h_{i}$, shown in Fig. 2(c) - as well as that of the real symmetrical components of the QGT, shown in the Supplemental Materialreveals that the distribution of the QGT is not perfectly O(3) symmetrical, and slightly breaks rotational symmetry down to a cubic symmetry even at the critical point. This small symmetry breaking is equally present in random and homogeneous fields; therefore it cannot be induced by the direction of the magnetic field [41], which could anyway only explain the emergence of a tetragonal symmetry. Rather, we explain this behavior as an effect of the cubic shape of the system on the structure of critical wave functions.

Universal Hall conductance fluctuations.-The behavior of the QGT at the critical point has an interesting experimental consequence. The antisymmetric part of the QGT is directly related to the Hall conductance through the Kubo-Greenwood formula [40,59],

$$
G_{H}^{k}=\frac{e^{2}}{\hbar} \sum_{E_{\alpha}<E_{\mathrm{F}}} h_{k}(\alpha),
$$

with $E_{\mathrm{F}}$ as the Fermi-energy, and $k \in\{x, y, z\}$ as the direction perpendicular to the plane of the Hall measurement. According to Eq. (8), chemical potential changes in a coherent mesoscopic sample induce Hall conductance fluctuations, determined by the critical distribution of the QGT. Consequently, in a magnetic field, at the metal-insulator transition, we predict the emergence of universal and isotropic Hall conductance fluctuations in a mesoscopic sample. These fluctuations as well as their precise distributions should be accessible in present-day experiments. As a possible implementation, one can think of strongly disordered metallic samples, similar to those of Ref. [60] and in a Hall-measurement setup [61], with the mobility edge tuned, e.g., by applying strain. Changing the external magnetic field or the application of back gates should generate the mesoscopic fluctuations discussed here [60,61].

Conclusions.-Our results show that the quantum geometrical structure of the eigenstates is intimately related to 
the conductance properties at the Fermi energy. The QGT displays universal scaling and distribution properties, and successfully captures the crossover between orthogonal and unitary Anderson-localized critical states. A natural generalization would be to study the behavior of QGT in models with weak spin-orbit coupling $[53,62]$. In that case-in the presence of time reversal symmetry-one expects a two parameter crossover between the orthogonal and the symplectic classes. If both spin-orbit coupling and magnetic fields are present, an even more complicated, three parameter behavior may appear. Generalizations in the presence of interaction and for the many body localization (MBL) transition are other open lines of research $[63,64]$, though the extremely limited system sizes make the scaling analysis of the QGT at the MBL transition significantly harder.

We are grateful to Nigel Cooper and Bert Halperin for illuminating discussions. This research has been supported by the Hungarian National Research, Development and Innovation Office (NKFIH) through Grant No. SNN118028 and the Hungarian Quantum Technology National Excellence Program (Project No. 2017-1.2.1-NKP2017-00001). M. A. W. has been supported by the ÚNKP17-3-IV New National Excellence Program of the Ministry of Human Capacities. This work was partially supported by the Research Council of Norway through its Centres of Excellence funding scheme, Project No. 262633 "QuSpin." F. v. O. was supported in part by CRC 183 of the Deutsche Forschungsgemeinschaft.

[1] J. P. Provost and G. Vallee, Commun. Math. Phys. 76, 289 (1980).

[2] R. A. Fisher, Proc. Cambridge Philos. Soc. 22, 700 (1925).

[3] M. A. Nielsen and I. L. Chuang, Quantum Computation and Quantum Information (Cambridge University Press, Cambridge, England, 2000), pp. 403-416.

[4] P. Zanardi, P. Giorda, and M. Cozzini, Phys. Rev. Lett. 99, 100603 (2007).

[5] M. Kolodrubetz, V. Gritsev, and A. Polkovnikov, Phys. Rev. B 88, 064304 (2013).

[6] P. Zanardi and N. Paunkovic, Phys. Rev. E 74, 031123 (2006)

[7] S. Garnerone, N. T. Jacobson, S. Haas, and P. Zanardi, Phys. Rev. Lett. 102, 057205 (2009).

[8] P. Buonsante and A. Vezzani, Phys. Rev. Lett. 98, 110601 (2007).

[9] J. Carrasquilla, S. R. Manmana, and M. Rigol, Phys. Rev. A 87, 043606 (2013).

[10] S. Yang, S.-J. Gu, C.-P. Sun, and H.-Q. Lin, Phys. Rev. A 78, 012304 (2008).

[11] S. Garnerone, D. Abasto, S. Haas, and P. Zanardi, Phys. Rev. A 79, 032302 (2009).

[12] C. De Grandi, A. Polkovnikov, and A. W. Sandvik, Phys. Rev. B 84, 224303 (2011).

[13] M. V. Berry, Proc. R. Soc. A 392, 45 (1984).

[14] Y. Aharonov and D. Bohm, Phys. Rev. 115, 485 (1959).
[15] M. Atala, M. Aidelsburger, J. T. Barreiro, D. Abanin, T. Kitagawa, E. Demler, and I. Bloch, Nat. Phys. 9, 795 (2013).

[16] G. Sundaram and Q. Niu, Phys. Rev. B 59, 14915 (1999).

[17] K. Y. Bliokh, A. Niv, V. Kleiner, and E. Hasman, Nat. Photonics 2, 748 (2008).

[18] J. Zak, Phys. Rev. Lett. 62, 2747 (1989).

[19] M. Z. Hasan and C. L. Kane, Rev. Mod. Phys. 82, 3045 (2010).

[20] M. Hafezi, S. Mittal, J. Fan, A. Migdall, and J. M. Taylor, Nat. Photonics 7, 1001 (2013).

[21] C.-Z. Chang et al., Science 340, 167 (2013).

[22] J. Anandan, Phys. Lett. 133A, 171 (1988).

[23] P. San-Jose, G. Zaránd, A. Shnirman, and G. Schön, Phys. Rev. Lett. 97, 076803 (2006).

[24] J. Alicea, Y. Oreg, G. Refael, F. von Oppen, and M. P. A. Fisher, Nat. Phys. 7, 412 (2011).

[25] L. Campos Venuti and P. Zanardi, Phys. Rev. Lett. 99, 095701 (2007).

[26] P. W. Anderson, Phys. Rev. 109, 1492 (1958).

[27] B. Kramer and A. MacKinnon, Rep. Prog. Phys. 56, 1469 (1993).

[28] M. A. Werner, A. Brataas, F. von Oppen, and G. Zarand, Phys. Rev. B 91, 125418 (2015).

[29] E. Abrahams, P. W. Anderson, D. C. Licciardello, and T. V. Ramakrishnan, Phys. Rev. Lett. 42, 673 (1979).

[30] A. MacKinnon and B. Kramer, Phys. Rev. Lett. 47, 1546 (1981).

[31] K. Slevin and T. Ohtsuki, New J. Phys. 16, 015012 (2014).

[32] K. Slevin and T. Ohtsuki, Phys. Rev. Lett. 78, 4083 (1997).

[33] D. E. Khmel'nitskii and A. I. Larkin, Solid State Commun. 39, 1069 (1981).

[34] G. Zaránd, C. P. Moca, and B. Jankó, Phys. Rev. Lett. 94, 247202 (2005).

[35] F. Wegner, Nucl. Phys. B270, 1 (1986).

[36] X.-F. Wang, Z. Wang, G. Kotliar, and C. Castellani, Phys. Rev. Lett. 68, 2504 (1992).

[37] A. M. M. Pruisken, Physica (Amsterdam) 181A, 1 (1992).

[38] M. Batsch, L. Schweitzer, I. Kh. Zharekeshev, and B. Kramer, Phys. Rev. Lett. 77, 1552 (1996).

[39] J. T. Edwards and D. J. Thouless, J. Phys. C 5, 807 (1972).

[40] D. J. Thouless, M. Kohmoto, M. P. Nightingale, and M. den Nijs, Phys. Rev. Lett. 49, 405 (1982).

[41] See Supplemental Material at http://link.aps.org/ supplemental/10.1103/PhysRevLett.122.106601 for methodological details and additional data, which includes Refs. [42-46].

[42] M. Kolodrubetz, D. Sels, P. Mehta, and A. Polkovnikov, Phys. Rep. 697, 1 (2017).

[43] D. Braun, E. Hofstetter, G. Montambaux, and A. MacKinnon, Phys. Rev. B 55, 7557 (1997).

[44] R. Christensen, Advanced Linear Modeling (Springer-Verlag, New York, 2001), 2nd ed.

[45] B. I. Shklovskii, B. Shapiro, B. R. Sears, P. Lambrianides, and H. B. Shore, Phys. Rev. B 47, 11487 (1993).

[46] C. M. Canali, C. Basu, W. Stephan, and V. E. Kravtsov, Phys. Rev. B 54, 1431 (1996).

[47] P. W. Anderson, D. J. Thouless, E. Abrahams, and D. S. Fisher, Phys. Rev. B 22, 3519 (1980).

[48] F. Evers and A. D. Mirlin, Phys. Rev. Lett. 84, 3690 (2000). 
[49] L. J. Vasquez, A. Rodriguez, and R. A. Römer, Phys. Rev. B 78, 195106 (2008).

[50] A. M. M. Pruisken, Phys. Rev. Lett. 61, 1297 (1988).

[51] D. E. Khmelnitskii, JETP Lett. 38, 552 (1983).

[52] T. Morimoto and H. Aoki, Phys. Rev. B 85, 165445 (2012).

[53] L. Ujfalusi and I. Varga, Phys. Rev. B 91, 184206 (2015).

[54] K. G. Wilson, Rev. Mod. Phys. 47, 773 (1975).

[55] This dimension just follows from the requirement that, at the corresponding magnetic length scale $L_{\text {flux }}$, the random flux be of size $h c / e$. Notice that this is not the dimension of the homogeneous magnetic field, $y_{\text {hom }}=2$, following from gauge invariance $[33,36]$.

[56] F. von Oppen, Phys. Rev. Lett. 73, 798 (1994).
[57] F. von Oppen, Phys. Rev. E 51, 2647 (1995).

[58] B. Shapiro, Phys. Rev. Lett. 65, 1510 (1990).

[59] D. A. Greenwood, Proc. Phys. Soc. 71, 585 (1958).

[60] H. T. Mebrahtu, I. V. Borzenets, H. Zheng, Y. V. Bomze, A. I. Smirnov, S. Florens, H. U. Baranger, and G. Finkelstein, Nat. Phys. 9, 732 (2013).

[61] F. Haas, P. Zellekens, M. Lepsa, T. Rieger, D. Grützmacher, H. Lüth, and T. Schäpers, Nano Lett. 17, 128 (2017).

[62] T. Kawarabayashi, T. Ohtsuki, K. Slevin, and Y. Ono, Phys. Rev. Lett. 77, 3593 (1996).

[63] A. Pal and D. A. Huse, Phys. Rev. B 82, 174411 (2010).

[64] M. Filippone, P. W. Brouwer, J. Eisert, and F. von Oppen, Phys. Rev. B 94, 201112(R) (2016). 